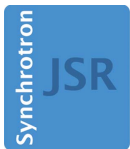

JOURNAL OF SYNCHROTRON RADIATION

Received 6 August 2019

Accepted 7 October 2019

Edited by I. Schlichting, Max Planck Institute for Medical Research, Germany

Keywords: XEOL; TR-XEOL; XRF; streak camera.

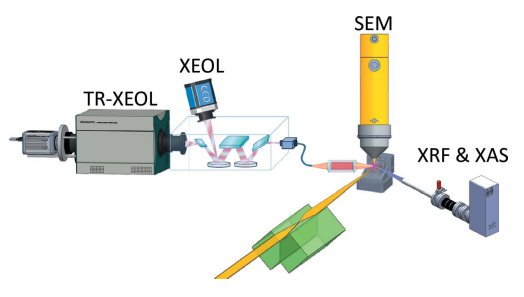

C 2020 International Union of Crystallography

\section{Capabilities of time-resolved X-ray excited optical luminescence of the Taiwan Photon Source 23A $X$-ray nanoprobe beamline}

\author{
Bi-Hsuan Lin, ${ }^{a} *$ Yu-Hao Wu, ${ }^{a}$ Xiao-Yun Li, ${ }^{a}$ Hsu-Cheng Hsu, ${ }^{b}$ Yu-Cheng Chiu, ${ }^{c}$ \\ Chien-Yu Lee, ${ }^{a}$ Bo-Yi Chen, ${ }^{a}$ Gung-Chian Yin, ${ }^{a}$ Shao-Chin Tseng, ${ }^{a}$ \\ Shih-Hung Chang, ${ }^{a}$ Mau-Tsu Tang ${ }^{a}$ and Wen-Feng Hsieh ${ }^{d}$
}

\begin{abstract}
${ }^{\mathbf{a}}$ National Synchrotron Radiation Research Center, Hsinchu 30076, Taiwan, ${ }^{\mathbf{b}}$ Department of Photonics, National Cheng Kung University, Tainan 70101, Taiwan, '⿳一 Department of Chemical Engineering, National Taiwan University of Science and Technology, Taipei 10607, Taiwan, and ${ }^{\mathbf{d}}$ Department of Photonics and Institute of Electro-Optical Engineering, National Chiao Tung University, Hsinchu 30010, Taiwan. *Correspondence e-mail: bihsuan@nsrrc.org.tw
\end{abstract}

Time-resolved X-ray excited optical luminescence (TR-XEOL) was developed successfully for the 23A X-ray nanoprobe beamline located at the Taiwan Photon Source (TPS). The advantages of the TR-XEOL facility include (i) a nano-focused X-ray beam $(<60 \mathrm{~nm})$ with excellent spatial resolution and (ii) a streak camera that can simultaneously record the XEOL spectrum and decay time. Three time spans, including normal ( 30 ps to $2 \mathrm{~ns}$ ), hybrid ( 30 ps to $310 \mathrm{~ns}$ ) and single ( $30 \mathrm{ps}$ to $1.72 \mu \mathrm{s}$ ) bunch modes, are available at the TPS, which can fulfil different experimental conditions involving samples with various lifetimes. It is anticipated that TR-XEOL at the TPS X-ray nanoprobe could provide great characterization capabilities for investigating the dynamics of photonic materials.

\section{Introduction}

X-ray excited optical luminescence (XEOL) (Nie et al., 2017; Ma et al., 2019; Novais et al., 2012; Salomon et al., 2017) is a well developed and advanced characterization technique employed at synchrotron radiation facilities. Although this method involves a relatively simple X-ray-photon-in/opticalphoton-out measurement, XEOL is a promising technique for investigating optical decay channels and their relationship with specific absorption processes at the core level (Sham et al., 1993; Ward et al., 2013; Heigl et al., 2007). In synchrotron source pumping, the X-ray energy can be tuned across the absorption edge of a specific element of the sample to study its emission properties. According to the report of Sham \& Rosenberg (2007) the tunable synchrotron source can create an excited state to decay optically via more pathways through exciting the core-to-continuum, core-to-quasi-bound and core-to-bound transitions. Using the high-energy resolution of the synchrotron source, different chemical sites or polytypes of the same element can also be sensitive to a specific channel via selective excitation. Time-resolved XEOL (TR-XEOL) could be developed to investigate the luminescence dynamic process of materials, as the synchrotron source offers the advantage of good temporal domain measurements. TRXEOL can be performed at several synchrotron facilities, including the Canadian Light Source (CLS) (Sham, 2014; Armelao et al., 2010), European Synchrotron Radiation Facility (ESRF) (Martínez-Criado et al., 2014), Advanced Photon Source (APS) (Heigl et al., 2007; Sham \& Rosenberg, 2007), Cornell High Energy Synchrotron Source (CHESS) 
(O'Malley et al., 2011) and Diamond Light Source (DLS) (Taylor et al., 2013; Mosselmans et al., 2013). Based on the requirements of previous research studies, a TR-XEOL facility was created for the 23A X-ray nanoprobe beamline located at the Taiwan Photon Source (TPS) at the National Synchrotron Radiation Research Center of Taiwan. The pulse duration of the TPS is $\sim 30 \mathrm{ps}$, which was measured by Liao et al. (2015). Three different filling patterns of the electron bunches can be applied at the TPS: single, normal and hybrid bunch modes, which provide time scales of $30 \mathrm{ps}$ to $1.72 \mu \mathrm{s}$, $30 \mathrm{ps}$ to $2 \mathrm{~ns}$ and $30 \mathrm{ps}$ to $310 \mathrm{~ns}$, respectively. Thus, a suitable time scale can be selected based on the desired TR-XEOL experiments. In this report, the capabilities of TR-XEOL at the TPS 23A beamline were evaluated using three test samples, i.e. trihalide perovskite $\mathrm{CH}_{3} \mathrm{NH}_{3} \mathrm{PbBr}_{3}$ (Kumar et al., 2016), a single $\mathrm{ZnO}$ microrod (Lin et al., 2019) and a $c$-plane $\mathrm{ZnO}$ wafer (Lin et al., 2016), to demonstrate TR-XEOL operation in the single, normal and hybrid bunch modes, respectively. Furthermore, the advantages of using the streak camera as a detector will also be discussed.

\section{Experimental setup and results}

\subsection{Endstation functions}

The TPS 23A X-ray nanoprobe is a multifunctional hard $\mathrm{X}$-ray $(4-15 \mathrm{keV})$ beamline. Fig. 1 shows a schematic of the experimental setup and associated functions. The X-ray beam was focused using Montel mirrors (Ice et al., 2011; Yin et al., 2016), which can deliver X-ray beam spot sizes of less than $60 \mathrm{~nm}$. However, according to results from Rogalev \& Goulon (2002), the spatial resolution at these X-ray energies will be limited by secondary excitations created by the X-rays to 150 $200 \mathrm{~nm}$. The sample was mounted on a piezo flexure stage with fine and coarse alignments, which provide traveling ranges of $100 \mu \mathrm{m}$ and $25 \mathrm{~mm}$, respectively. A three-axis laser interferometer was used to precisely monitor the position of the sample stage in the 2D mapping measurements with on-the-fly scanning (Lee et al., 2018). X-ray absorption spectroscopy

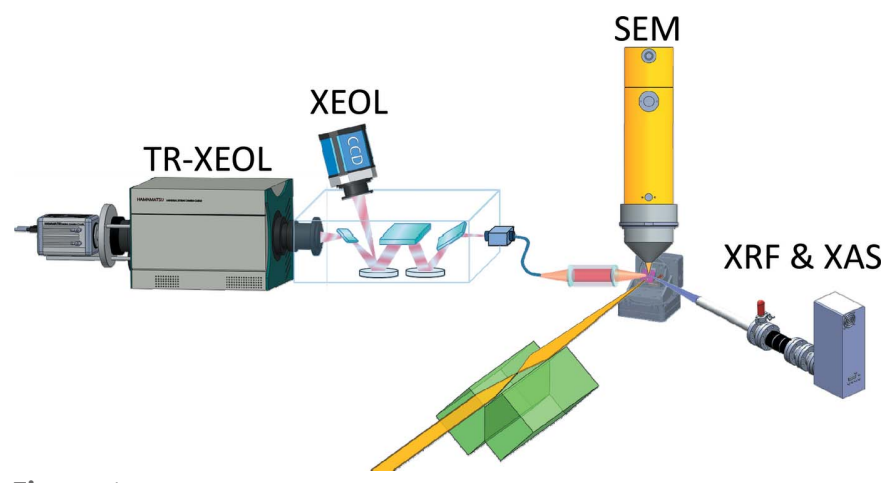

Figure 1

The main functions of the X-ray nanoprobe at the TPS 23A beamline located at the Taiwan Photon Source, including XEOL, TR-XEOL, XRF, $\mathrm{XAS}$ and CL. The measured sample will be equipped in the environment of a high vacuum $\left(1 \times 10^{-6}\right.$ torr $)$ and room temperature $(300 \mathrm{~K})$ with a load-lock system to quickly transfer the samples between the main chamber and the preparation chamber.
(XAS) and X-ray fluorescence (XRF) were conducted using a silicon drift detector (SDD; Vortex-ME4, HITACHI). The TR-XEOL was collected by a $10 \mathrm{~m}$ fiber and directed to a spectrometer (iHR320, HORIBA) equipped with a Hamamatsu C10910 streak camera and M10913 slow single sweep unit. A Stanford Research Systems DG645 delay generator was used, operating with a jitter of $\sim 25$ ps. Typical image magnifications for input optics, streak tube and output optics of the streak camera are 1:1, 1:2.8 and 1:0.7, respectively. The CCD of the streak camera has 1344 (horizontal for wavelength) $\times 1024$ (vertical for time) pixels with a cell size of $6.45 \mu \mathrm{m} \times 6.45 \mu \mathrm{m}$. In this case, for a streak image with $2 \mathrm{~ns}$ sweep time and with the streak slit opened to $40 \mu \mathrm{m}$, we calculated the related resolution to be about 24 ps for the detector. Concerning the resolution of the TR-XEOL, one should consider the instrument response function (IRF). The IRF has three main factors: the pulsed duration times of the source $\left(\tau_{\mathrm{s}}\right)$, the electronics $\left(\tau_{\mathrm{e}}\right)$ and the detector $\left(\tau_{\mathrm{d}}\right)$, and can be approximated as IRF $\cong\left[\left(\tau_{\mathrm{s}}\right)^{2}+\left(\tau_{\mathrm{e}}\right)^{2}+\left(\tau_{\mathrm{d}}\right)^{2}\right]^{1 / 2}$. We can then calculate the IRF to be $45 \mathrm{ps}$, using $\tau_{\mathrm{s}} \cong 30 \mathrm{ps}, \tau_{\mathrm{e}} \cong 25 \mathrm{ps}$ and $\tau_{\mathrm{d}} \cong 24 \mathrm{ps}$ in the case of a streak image with $2 \mathrm{~ns}$ sweep time.

The XEOL was also collected by an optical fiber and directed to another spectrometer (iHR550, HORIBA) with a liquid-nitrogen-cooled charge-coupled device (CCD; BIUV Symphony II) with a resolution of $2048 \times 512$ pixels. The iHR550 spectrometer was equipped with a photomultiplier tube (PMT) and switching mirror, which could be used to perform XEOL mapping. The collected emission wavelengths of both TR-XEOL and XEOL ranged from $200 \mathrm{~nm}$ to $1000 \mathrm{~nm}$. A scanning electron microscope (SEM) was also installed at the endstation to quickly identify sample positions. Additionally, the SEM could also be used for scanning cathodoluminescence $(\mathrm{CL})$ as a complementary method to analyze the emission properties of materials (Lin et al., 2019).

\subsection{Single bunch mode}

Fig. 2 shows the capabilities of XRF and XEOL mapping with $13.5 \mathrm{keV} \mathrm{X}$-rays (above the $\mathrm{Br} K$-edge and $\mathrm{Pb} L_{3}$-edge at $13.474 \mathrm{keV}$ and $11.035 \mathrm{keV}$, respectively). The elemental distributions of the trihalide perovskite $\mathrm{CH}_{3} \mathrm{NH}_{3} \mathrm{PbBr}_{3}$ corresponding to $\mathrm{Pb}$ and $\mathrm{Br}$ are shown in Figs. 2(a) and 2(b), respectively, while the XEOL spectrum and maps of the related emissions are shown in Figs. 2(c) and 2(d). The XEOL spectrum indicates that the main emission peak had a wavelength of $\sim 535 \mathrm{~nm}$. The results clearly show the elemental distributions and their corresponding emission peaks. The XRF and XEOL maps could be measured simultaneously in one scan. All emission lines under $13.5 \mathrm{keV}$ were recorded by a silicon drift detector and plotted using the beamline software (Lee et al., 2018).

Luminescence dynamics of the trihalide perovskite $\mathrm{CH}_{3} \mathrm{NH}_{3} \mathrm{PbBr}_{3}$ were measured using TR-XEOL in the single bunch mode, as shown in Fig. 3. The TPS has a circumference of $\sim 518 \mathrm{~m}$, with an available time scale of $\sim 1.72 \mu \mathrm{s}(578 \mathrm{kHz})$ in the single bunch mode and beam current of $2 \mathrm{~mA}$. Thus, the 

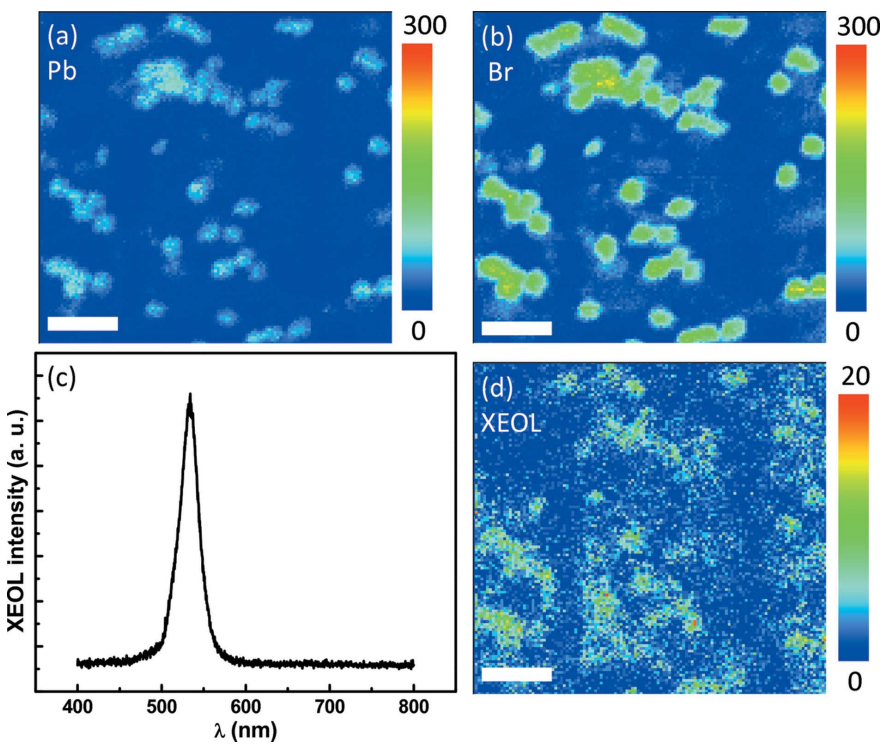

Figure 2

$\mathrm{X}$-ray fluorescence maps of elements $(a) \mathrm{Pb}$ and $(b) \mathrm{Br}$. The X-ray excited optical luminescence (XEOL) spectrum and perovskite map are shown in $(c)$ and $(d)$, respectively. All scale bars correspond to $10 \mu \mathrm{m}$.

repetition rate of the M10913 slow single sweep unit was set to $578 \mathrm{kHz}$ in the external rising mode in the TR-XEOL experiments. Fig. 3(a) shows the streak image recorded with a $10 \mu$ sweep time, while its spectral integrated $(510-530 \mathrm{~nm})$ time trace is shown in Fig. 3(b). Five repeated risings can be observed where the interval time of each rising was $1.72 \mu \mathrm{s}$. This clearly demonstrates that the electron filling pattern was operated in the single bunch mode. The sweep time of the streak image can be tuned to a suitable range, for example to $500 \mathrm{~ns}$ as shown in Fig. 3(c) for the TR-XEOL measurements. The spectral integrated $(510-530 \mathrm{~nm})$ time traces were fitted using two exponential decay functions, as shown in Fig. $3(d)$, to obtain decay lifetimes of $\tau_{1} \simeq$ $6 \mathrm{~ns}$ and $\tau_{2} \simeq 64 \mathrm{~ns}$.

\subsection{Normal bunch mode}

As the electron RF-cavity of the TPS was operated at $500 \mathrm{MHz}$ (2 ns), the normal bunch mode could apply a time scale of $30 \mathrm{ps}$ to $2 \mathrm{~ns}$. Therefore, materials with a faster decay lifetime $(<2 \mathrm{~ns})$ could be measured using TR-XEOL at the TPS 23A beamline. The repetition frequency of TR-XEOL was set to $3.469 \mathrm{MHz}(499.665 \mathrm{MHz} / 144)$ in the normal bunch mode, as the maximal sweep repetition frequency of the M10913 slow single sweep unit was $4 \mathrm{MHz}$. In our previous report (Lin et al., 2019), we observed that the near band edge (NBE) emission intensity of a single $\mathrm{ZnO}$ microrod increased

Figure 4
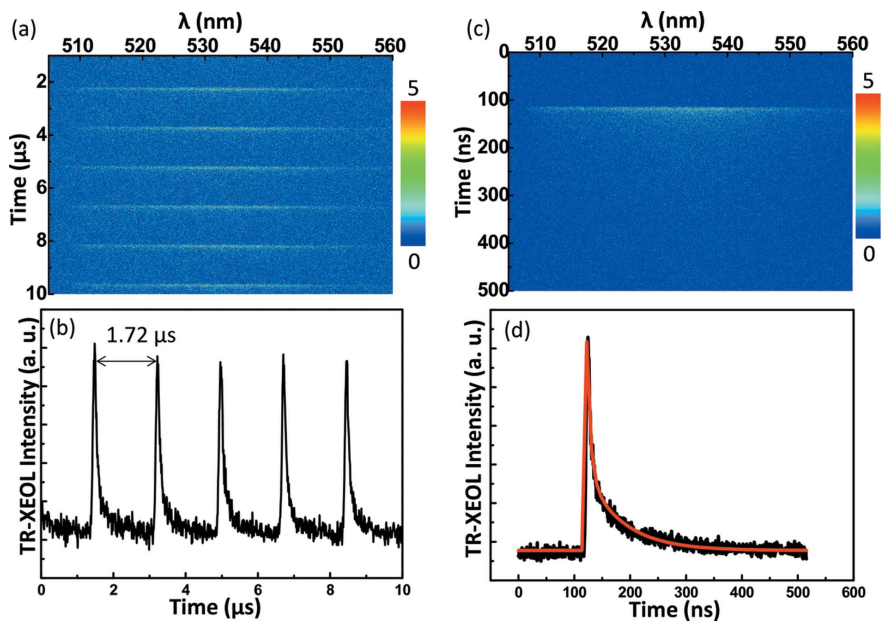

Figure 3

The streak image and corresponding spectral integrated time trace are shown in panels $(a)$ and $(b)$, respectively, obtained using a streak camera with a $10 \mu$ s time span, highlighting the $1.72 \mu$ s period of the single bunch mode. A $500 \mathrm{~ns}$ time span was used to obtain further information on the luminescence dynamics of the perovskite, creating the $(c)$ streak image and $(d)$ lifetime obtained by fitting the TR-XEOL trace.

nonlinearly with the XAS fluorescence yield. Thus, the luminescence dynamics of a single $\mathrm{ZnO}$ microrod can be investigated further using TR-XEOL. The single $\mathrm{ZnO}$ microrod and its Zn $K$-edge XAS spectra are shown in Figs. 4(a) and 4(b), respectively. This indicates the convenience of using SEM at the TPS 23A beamline to identify the sample quickly, and the advantages of using tunable and continuous X-ray energy set across the $\mathrm{Zn} K$-edge to study the luminescence dynamics. Figs. 4(c) and 4(d) show the streak images recorded with a 2 ns sweep time and the corresponding spectral integrated (360- (a)
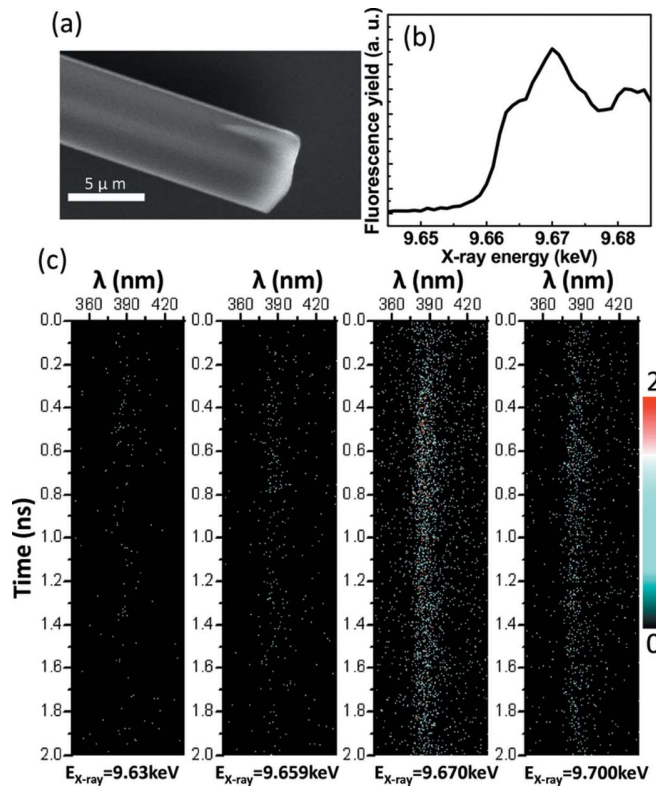
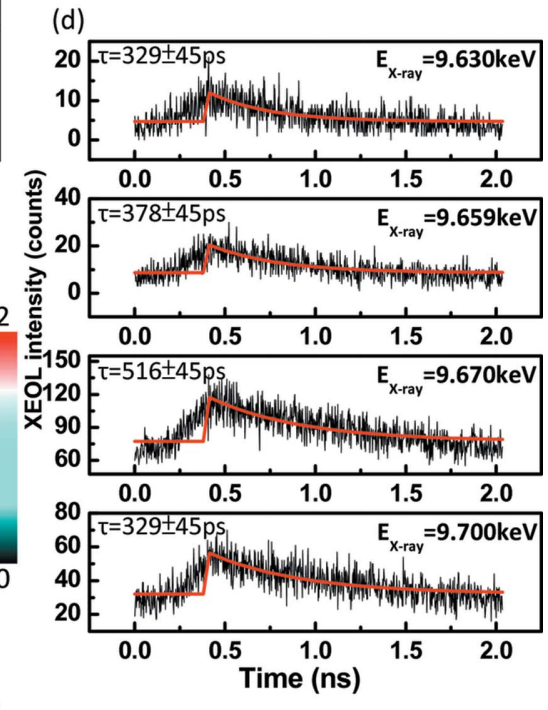

(a) SEM image and (b) Zn $K$-edge XAS for the single ZnO microrod. (c) The corresponding TRXEOL streak images with X-ray energies set across the $\mathrm{Zn} K$-edge and (d) the lifetimes obtained by fitting the TR-XEOL traces of $(c)$. 
$420 \mathrm{~nm}$ ) time traces of the single $\mathrm{ZnO}$ microrod. After fitting the spectral integrated time traces using a single exponential function, we obtained decay lifetimes of $329 \pm 45 \mathrm{ps}, 378 \pm$ $45 \mathrm{ps}, 516 \pm 45 \mathrm{ps}$ and $329 \pm 45 \mathrm{ps}$ for the $9.630 \mathrm{keV}, 9.659 \mathrm{keV}$, $9.670 \mathrm{keV}$ and $9.700 \mathrm{keV} \mathrm{X}$-ray energy excitations, respectively. It can be observed that the decay lifetime shows a similar positive edge jump following XAS across the $\mathrm{Zn} K$ edge. This indicates that the $\mathrm{XEOL}$ of the single $\mathrm{ZnO}$ microrod had the longest decay lifetime when pumped at the resonant energy $(9.670 \mathrm{keV})$ and shorter decay lifetimes when excited to levels either below or above the $\mathrm{Zn} K$-edge. We believe that this behavior was due to the high volumetric carrier densities caused by the pulsed X-rays at the resonant energy, which excited more free carriers compared with those excited below and above the $\mathrm{Zn} K$-edge energies. The peak power density of TPS 23A beamline is about $\sim 30 \mathrm{MW} \mathrm{cm}{ }^{-2}$ (repetition rate $500 \mathrm{MHz}, \mathrm{X}$-ray peak duration time $30 \mathrm{ps,}$ photon flux $10^{10}$ photons s $^{-1}$ and X-ray spot size $\sim 60 \mathrm{~nm}$ at $10 \mathrm{keV})$, which is about seven orders of magnitude larger than that of an unfocused $\mathrm{X}$-ray beam $\left(\sim 6 \mathrm{~W} \mathrm{~cm}^{-2}\right)$. This finding was consistent with our previous reports where the pulsed $\mathrm{X}$-rays excited the high-density low-energy free carriers at the resonant energy of the $\mathrm{Zn} K$-edge, resulting in Coulomb screening of the spontaneous polarization. This decreased the exciton-phonon interactions, causing a spectral blue shift of $40-50 \mathrm{meV}$ in the $\mathrm{ZnO}$ wafers (Lin et al., 2016, 2018). As the cooling of the hot carriers depended on interactions with the phonons, the reduced exciton-phonon interactions caused a slower decay of the free carriers.

On the other hand, the decay curve measurements of Fig. 4(d) show similar odd pre-excitation shoulders, which may be caused by chromatic dispersion from the optical fiber. TR$\mathrm{XEOL}$ is still in the early stage of development at the TPS 23A beamline; a more quantitative comparison with conventional methods requires further studies. A better data quality may be obtained by shortening the length of the optical fiber, and a good fitting technique will be learned from the fluorescence lifetime imaging community.

\subsection{Hybrid bunch mode}

The hybrid bunch mode was developed successfully at the TPS. The advantages of operating in the hybrid bunch mode are: (i) the electron beam current can reach $400 \mathrm{~mA}$, providing high X-ray photon flux, and (ii) the longer time interval can facilitate temporal domain measurements. There are $\sim 864$ electron bunches in the storage ring of the TPS, which have a minimum interval of $2 \mathrm{~ns}$. In our hybrid bunch mode, we could achieve $\sim 155$ symmetric electron bunches to obtain a $\sim 310 \mathrm{~ns}$ interval between the single and multi-electron bunches. Figs. 5( $a)$ and 5(b) show the streak images recorded with a $2 \mu$ s sweep time and the corresponding spectral integrated (380$400 \mathrm{~nm}$ ) time trace, respectively, of the $c$-plane $\mathrm{ZnO}$ wafer operating in the hybrid bunch mode. The time interval of the hybrid bunch mode was appropriate, as the decay lifetime of the $c$-plane $\mathrm{ZnO}$ was approximately hundreds of picoseconds. The streak image shown in Fig. 4(a) was measured at $578 \mathrm{kHz}$

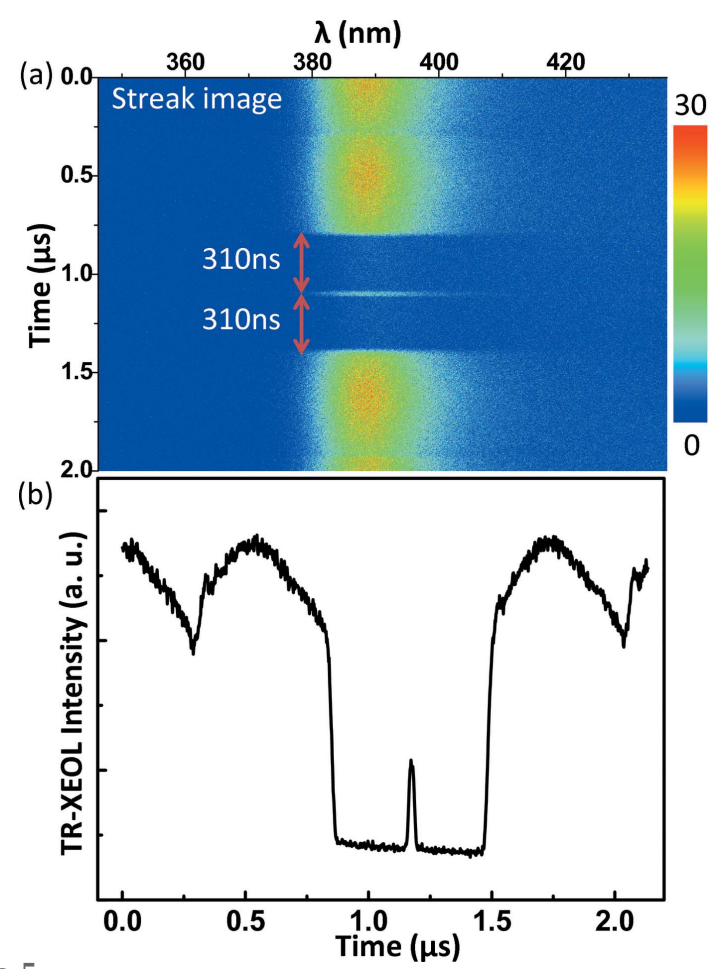

Figure 5

The streak image $(a)$ and spectral integrated time trace $(b)$ in the hybrid bunch mode.

(499.665 MHz/864), which could provide a time interval of $30 \mathrm{ps}$ to $310 \mathrm{~ns}$. The hybrid bunch mode still had electron bunches with 2 ns intervals, so a repetition rate of $3.469 \mathrm{MHz}$ (499.665 MHz/144) could also be used for time intervals of $30 \mathrm{ps}$ to $2 \mathrm{~ns}$, depending on user demand.

\section{Conclusions}

TR-XEOL capabilities were developed successfully for the TPS 23A beamline. The advantages of TR-XEOL include the following: (i) the nano-focused X-ray beam $(<60 \mathrm{~nm})$ offers excellent spatial resolution, and (ii) a streak camera can be employed to simultaneously record the XEOL emission spectrum and decay time. Three time intervals, including normal ( $30 \mathrm{ps}$ to $2 \mathrm{~ns}$ ), hybrid ( $30 \mathrm{ps}$ to $310 \mathrm{ns)}$ and single ( $30 \mathrm{ps}$ to $1.72 \mu \mathrm{s})$ bunch modes, are available to provide different experimental conditions to test samples with various emission lifetimes. The hybrid bunch, in particular, provides a high X-ray photon flux, which fulfils the requirements for a multi-functional beamline and the wide time spans necessary for taking temporal domain measurements. We anticipate that TR-XEOL at the TPS X-ray nanoprobe will offer powerful characterization capabilities for investigating the dynamics of photonic materials.

\section{Acknowledgements}

We would like to thank the National Synchrotron Radiation Research Center (NSRRC) and Ministry of Science and Technology of Taiwan for supporting this project. 


\section{Funding information}

The following funding is acknowledged: Ministry of Science and Technology of Taiwan (MOST 108-2112-M-213-006).

\section{References}

Armelao, L., Heigl, F., Brunet, S., Sammynaiken, R., Regier, T., Blyth, R. I., Zuin, L., Sankari, R., Vogt, J. \& Sham, T. K. (2010). ChemPhysChem, 11, 3625-3631.

Heigl, F., Jürgensen, A., Zhou, X.-T., Lam, S., Murphy, M., Ko, J. Y. P., Sham, T. K., Rosenberg, R. A., Gordon, R., Brewe, D., Regier, T. \& Armelao, L. (2007). AIP Conf. Proc. 879, 1202-1205.

Ice, G. E., Budai, J. D. \& Pang, J. W. L. (2011). Science, 334, 12341239.

Kumar, S., Jagielski, J., Yakunin, S., Rice, P., Chiu, Y.-C., Wang, M., Nedelcu, G., Kim, Y., Lin, S., Santos, E. J., Kovalenko, M. V. \& Shih, C. J. (2016). ACS Nano, 10, 9720-9729.

Lee, C.-Y., Yin, G.-C., Chen, B.-Y., Chen, H.-Y., Lin, B.-H., Tseng, S.-C., Chang, S.-H., Li, X.-Y. \& Tang, M.-T. (2018). Microsc. Microanal. 24, 106-107.

Liao, C.-Y., Wu, C.-Y., Cheng, Y.-S., Kuan, C.-K., Chuang, J.-Y., Hsueh, H.-P., Hsu, S.-Y., Hu, K. H. \& Hsu, K. T. (2015). Proceedings of the Sixth International Particle Accelerator Conference (IPAC2015), 3-8 May 2015, Richmond, VA, USA, pp. 11091111. MOPTY074.

Lin, B. H., Chen, H.-Y., Tseng, S.-C., Wu, J.-X., Chen, B.-Y., Lee, C.-Y., Yin, G.-C., Chang, S.-H., Tang, M.-T. \& Hsieh, W. F. (2016). Appl. Phys. Lett. 109, 192104.

Lin, B.-H., Li, X.-Y., Lin, D.-J., Jian, B.-L., Hsu, H.-C., Chen, H.-Y., Tseng, S.-C., Lee, C.-Y., Chen, B.-Y., Yin, G.-C., Hsu, M. Y., Chang, S. H., Tang, M. T. \& Hsieh, W. F. (2019). Sci. Rep. 9, 207.
Lin, B.-H., Wu, Y.-C., Chen, H.-Y., Tseng, S.-C., Wu, J.-X., Li, X.-Y., Chen, B.-Y., Lee, C.-Y., Yin, G.-C., Chang, S.-H., Tang, M.-T. \& Hsieh, W. F. (2018). Opt. Express, 26, 2731-2739.

Ma, J., Yao, Q., McLeod, J. A., Chang, L.-Y., Pao, C.-W., Chen, J., Sham, T.-K. \& Liu, L. (2019). Nanoscale, 11, 6182-6191.

Martínez-Criado, G., Segura-Ruiz, J., Alén, B., Eymery, J., Rogalev, A., Tucoulou, R. \& Homs, A. (2014). Adv. Mater. 26, 7873-7879.

Mosselmans, J., Taylor, R., Quinn, P., Finch, A., Cibin, G., Gianolio, D. \& Sapelkin, A. (2013). J. Phys. Conf. Ser. 425, 182009.

Nie, Y., Wang, Z., Wang, J., Bao, F., Zhang, J., Ma, Y., Sham, T.-K. \& Sun, X. (2017). J. Phys. Chem. C, 121, 26076-26085.

Novais, S. M. V., Valerio, M. E. G. \& Macedo, Z. S. (2012). J. Synchrotron Rad. 19, 591-595.

O'Malley, S. M., Revesz, P., Kazimirov, A. \& Sirenko, A. A. (2011). J. Appl. Phys. 109, 124906.

Rogalev, A. \& Goulon, J. (2002). Chemical Applications of Synchrotron Radiation, Part II: X-ray Applications, pp. 707-760. Singapore: World Scientific.

Salomon, D., Messanvi, A., Eymery, J. \& Martínez-Criado, G. (2017). Nano Lett. 17, 946-952.

Sham, T. K. (2014). Adv. Mater. 26, 7896-7901.

Sham, T. K., Jiang, D. T., Coulthard, I., Lorimer, J. W., Feng, X. H., Tan, K. H., Frigo, S. P., Rosenberg, R. A., Houghton, D. C. \& Bryskiewicz, B. (1993). Nature, 363, 331-334.

Sham, T. K. \& Rosenberg, R. A. (2007). ChemPhysChem, 8, 25572567.

Taylor, R. P., Finch, A. A., Mosselmans, J. F. W. \& Quinn, P. D. (2013). J. Lumin. 134, 49-58.

Ward, M. J., Regier, T. Z., Vogt, J. M., Gordon, R. A., Han, W. Q. \& Sham, T. K. (2013). J. Phys. Conf. Ser. 425, 092006.

Yin, G.-C., Chang, S.-H., Chen, B.-Y., Chen, H.-Y., Lin, B.-H., Tseng, S.-C., Lee, C.-Y., Wu, J.-X., Wu, S.-Y. \& Tang, M.-T. (2016). AIP Conf. Proc. 1741, 030004. 